\title{
Entropic bounds and continual measurements
}

\author{
Alberto Barchielli \\ Politecnico di Milano, Dipartimento di Matematica, \\ Piazza Leonardo da Vinci 32, I-20133 Milano, Italy. \\ E-mail: Alberto.Barchielli@polimi.it \\ Giancarlo Lupieri \\ Università degli Studi di Milano, Dipartimento di Fisica, \\ Via Celoria 16, I-20133 Milano, Italy. \\ E-mail: Giancarlo.Lupieri@mi.infn.it
}

November 3, 2005

\begin{abstract}
Some bounds on the entropic informational quantities related to a quantum continual measurement are obtained and the time dependencies of these quantities are studied.
\end{abstract}

\section{Introduction}

In the problem of information transmission through quantum systems, various entropic quantities appear which characterize the performances of the encoding and decoding apparatuses. Due to the peculiar character of a quantum measurement, many bounds on the informational quantities involved have been proved to hold [1-8]. In the case of measurements continual in time, these bounds acquire new aspects (family of measurements are now involved) and new problems arise. A typical question is about which of the various entropic measures of information is monotonically increasing or decreasing in time. We already started the study of this subject in Refs. [9,10]; here we apply to the case of continual measurements the new techniques developed [6-8] for the time independent case.

\subsection{Notations and preliminaries}

We denote by $\mathcal{L}(\mathcal{A} ; \mathcal{B})$ the space of bounded linear operators from $\mathcal{A}$ to $\mathcal{B}$, where $\mathcal{A}, \mathcal{B}$ are Banach spaces; moreover we set $\mathcal{L}(\mathcal{A}):=\mathcal{L}(\mathcal{A} ; \mathcal{A})$.

Let $\mathcal{H}$ be a separable complex Hilbert space; a normal state on $\mathcal{L}(\mathcal{H})$ is identified with a statistical operator, $\mathcal{T}(\mathcal{H})$ and $\mathcal{S}(\mathcal{H}) \subset \mathcal{T}(\mathcal{H})$ are the traceclass and the space of the statistical operators on $\mathcal{H}$, respectively, and $\|\rho\|_{1}:=$ $\operatorname{Tr} \sqrt{\rho^{*} \rho},\langle\rho, a\rangle:=\operatorname{Tr}_{\mathcal{H}}\{\rho a\}, \rho \in \mathcal{T}(\mathcal{H}), a \in \mathcal{L}(\mathcal{H})$. 
More generally, if $a$ belongs to a $W^{*}$-algebra and $\rho$ to its dual $\mathcal{M}^{*}$ or predual $\mathcal{M}_{*}$, the functional $\rho$ applied to $a$ is denoted by $\langle\rho, a\rangle$.

\subsubsection{A quantum/classical algebra}

Let $(\Omega, \mathcal{F}, Q)$ be a measure space, where $Q$ is a $\sigma$-finite measure. By Theorem 1.22 .13 of [11], the $W^{*}$-algebra $L^{\infty}(\Omega, \mathcal{F}, Q) \otimes \mathcal{L}(\mathcal{H})\left(W^{*}\right.$-tensor product) is naturally isomorphic to the $W^{*}$-algebra $L^{\infty}(\Omega, \mathcal{F}, Q ; \mathcal{L}(\mathcal{H}))$ of all the $\mathcal{L}(\mathcal{H})$ valued $Q$-essentially bounded weakly* measurable functions on $\Omega$. Moreover ( [11], Proposition 1.22.12), the predual of this $W^{*}$-algebra is $L^{1}(\Omega, \mathcal{F}, Q ; \mathcal{T}(\mathcal{H}))$, the Banach space of all the $\mathcal{T}(\mathcal{H})$-valued Bochner $Q$-integrable functions on $\Omega$, and this predual is naturally isomorphic to $L^{1}(\Omega, \mathcal{F}, Q) \otimes \mathcal{T}(\mathcal{H})$ (tensor product with respect to the greatest cross norm - [11], pp. $45,58,59,67,68)$.

Let us note that a normal state $\sigma$ on $L^{\infty}(\Omega, \mathcal{F}, Q ; \mathcal{L}(\mathcal{H}))$ is a measurable function $\omega \mapsto \sigma(\omega) \in \mathcal{T}(\mathcal{H}), \sigma(\omega) \geq 0$, such that $\operatorname{Tr}_{\mathcal{H}}\{\sigma(\omega)\}$ is a probability density with respect to $Q$.

\subsection{Quantum channels and entropies}

\subsubsection{Relative and mutual entropies}

The general definition of the relative entropy $S(\Sigma \mid \Pi)$ for two states $\Sigma$ and $\Pi$ is given in [12]; here we give only some particular cases of the general definition.

Let us consider two quantum states $\sigma, \tau \in \mathcal{S}(\mathcal{H})$ and two classical states $q_{k}$ on $L^{\infty}(\Omega, \mathcal{F}, Q)$ (two probability densities with respect to $Q$ ). The quantum relative entropy and the classical one are

$$
\begin{aligned}
S_{\mathrm{q}}(\sigma \mid \tau) & =\operatorname{Tr}_{\mathcal{H}}\{\sigma(\log \sigma-\log \tau)\}, \\
S_{\mathrm{c}}\left(q_{1} \mid q_{2}\right) & =\int_{\Omega} Q(\mathrm{~d} \omega) q_{1}(\omega) \log \frac{q_{1}(\omega)}{q_{2}(\omega)} .
\end{aligned}
$$

We shall need also the von Neumann entropy of a state $\tau \in \mathcal{S}(\mathcal{H}): S_{\mathrm{q}}(\tau):=$ $-\operatorname{Tr}\{\tau \log \tau\}$.

Let us consider now two normal states $\sigma_{k}$ on $L^{\infty}(\Omega, \mathcal{F}, Q ; \mathcal{L}(\mathcal{H}))$ and set $q_{k}(\omega):=\operatorname{Tr}\left\{\sigma_{k}(\omega)\right\}, \varrho_{k}(\omega):=\sigma_{k}(\omega) / q_{k}(\omega)$ (these definitions hold where the denominators do not vanish and are completed arbitrarily where the denominators vanish). Then, the relative entropy is

$$
\begin{aligned}
S\left(\sigma_{1} \mid \sigma_{2}\right) & =\int_{\Omega} Q(\mathrm{~d} \omega) \operatorname{Tr}_{\mathcal{H}}\left\{\sigma_{1}(\omega)\left(\log \sigma_{1}(\omega)-\log \sigma_{2}(\omega)\right)\right\} \\
& =S_{\mathrm{c}}\left(q_{1} \mid q_{2}\right)+\int_{\Omega} Q(\mathrm{~d} \omega) q_{1}(\omega) S_{\mathrm{q}}\left(\varrho_{1}(\omega) \mid \varrho_{2}(\omega)\right) .
\end{aligned}
$$

We are using a subscript "c" for classical entropies, a subscript "q" for purely quantum ones and no subscript for general entropies, eventually of a mixed character.

Classically a mutual entropy is the relative entropy of a joint probability with respect to the product of its marginals and this key notion can be generalized immediately to states on von Neumann algebras, every times we have a state on a tensor product of algebras [6-8]. 


\subsubsection{Channels}

Definition 1. ( [12] p. 137) Let $\mathcal{M}_{1}$ and $\mathcal{M}_{2}$ be two $W^{*}$-algebras. A linear map $\Lambda^{*}$ from $\mathcal{M}_{2}$ to $\mathcal{M}_{1}$ is said to be a channel if it is completely positive, unital (i.e. identity preserving) and normal (or, equivalently, weakly* continuous).

Due to the equivalence [13] of $\mathrm{w}^{*}$-continuity and existence of a preadjoint $\Lambda$, a channel is equivalently defined by: $\Lambda$ is a completely positive linear map from the predual $\mathcal{M}_{1 *}$ to the predual $\mathcal{M}_{2 *}$, normalized in the sense that $\left\langle\Lambda[\rho], \mathbb{1}_{2}\right\rangle_{2}=$ $\left\langle\rho, \mathbb{1}_{1}\right\rangle_{1}, \forall \rho \in \mathcal{M}_{1 *}$. Let us note also that $\Lambda$ maps normal states on $\mathcal{M}_{1}$ into normal states on $\mathcal{M}_{2}$.

A key result which follows from the convexity properties of the relative entropy is Uhlmann monotonicity theorem ( [12], Theor. 1.5 p. 21), which implies that channels decrease the relative entropy.

Theorem 1. If $\Sigma$ and $\Pi$ are two normal states on $\mathcal{M}_{1}$ and $\Lambda^{*}$ is a channel from $\mathcal{M}_{2} \rightarrow \mathcal{M}_{1}$, then $S(\Sigma \mid \Pi) \geq S(\Lambda[\Sigma] \mid \Lambda[\Pi])$.

\subsection{Continual measurements}

Let us axiomatize the properties of a probability space where an independentincrement process lives and that ones of the $\sigma$-algebras generated by its incre-

ments. The probability measure $Q_{1}$ we are introducing will play the role of a reference measure.

Assumption 1. Let $\left(X, \mathcal{X}, Q_{1}\right)$ be a probability space with $(X, \mathcal{X})$ standard Borel. Moreover:

1. $\left\{\mathcal{X}_{t}^{s}, 0 \leq s \leq t\right\}$ is a two-times filtration of sub- $\sigma$-algebras: $\mathcal{X}_{t}^{s} \subset \mathcal{X}_{T}^{r} \subset \mathcal{X}$ for $0 \leq r \leq s \leq t \leq T$;

2. $\forall t \geq 0, \mathcal{X}_{t}^{t}$ is trivial;

3. $\mathcal{X}_{t}^{s}=\bigwedge_{T: T>t} \mathcal{X}_{T}^{s}$ for $0 \leq s \leq t$;

4. $\mathcal{X}_{t}^{s}=\bigvee_{r: s<r<t} \mathcal{X}_{t}^{r}$ for $0 \leq s<t$;

5. $\mathcal{X}=\bigvee_{t: T>0} \mathcal{X}_{t}^{0}$;

6. for $0 \leq r \leq s \leq t \leq T, \mathcal{X}_{s}^{r}$ and $\mathcal{X}_{T}^{t}$ are $Q_{1}$-independent.

Continual measurements are a quantum analog of classical processes with independent increments $[10,14]$. As any kind of quantum measurement, a continual measurement is represented by instruments [15-17], but, as shown in [7], instruments are equivalent to particular types of channels. Here we introduce continual measurements directly as a family of channels satisfying a set of axioms (cf. also $[10,18])$.

Assumption 2. Let $\mathcal{H}$ be a separable complex Hilbert space. For all $s, t$, $0 \leq s \leq t$, we have a channel

$$
\tilde{\Lambda}_{t}^{s}: L^{1}\left(X, \mathcal{X}_{s}^{0}, Q_{1} ; \mathcal{T}(\mathcal{H})\right) \rightarrow L^{1}\left(X, \mathcal{X}_{t}^{0}, Q_{1} ; \mathcal{T}(\mathcal{H})\right)
$$

such that 
1. $\tilde{\Lambda}_{t}^{t}=11, t \geq 0$;

2. $\tilde{\Lambda}_{t}^{s} \circ \tilde{\Lambda}_{s}^{r}=\tilde{\Lambda}_{t}^{r}, 0 \leq r \leq s \leq t$;

3. $\forall \eta \in \mathcal{T}(\mathcal{H}), \tilde{\Lambda}_{t}^{s}[\eta]$ is $\mathcal{X}_{t}^{s}$-measurable, $0 \leq s \leq t$;

4. $\forall \eta \in \mathcal{T}(\mathcal{H}), \forall q \in L^{1}\left(X, \mathcal{X}_{s}^{0}, Q_{1}\right), \quad \tilde{\Lambda}_{t}^{s}[q \eta]=q \tilde{\Lambda}_{t}^{s}[\eta], \quad 0 \leq s \leq t$, (i.e. $\tilde{\Lambda}_{t}^{s}[q \eta](x)=q(x) \tilde{\Lambda}_{t}^{s}[\eta](x)$ a.s. $)$.

By points (3), (4) of Assumption 2 and (6) of Assumption 1 one gets: $\forall \sigma_{s} \in L^{1}\left(X, \mathcal{X}_{s}^{0}, Q_{1} ; \mathcal{T}(\mathcal{H})\right), 0 \leq s \leq t$,

$$
\mathbb{E}_{Q_{1}}\left[\tilde{\Lambda}_{t}^{s}\left[\sigma_{s}\right] \mid \mathcal{X}_{t}^{s}\right]=\tilde{\Lambda}_{t}^{s}\left[\mathbb{E}_{Q_{1}}\left[\sigma_{s}\right]\right] .
$$

Here $\mathbb{E}_{Q_{1}}$ and $\mathbb{E}_{Q_{1}}\left[\bullet \mid \mathcal{X}_{t}^{s}\right]$ are the classical expectation and conditional expectation extended to operator-valued random variable.

Let us also define the evolution

$$
\mathcal{U}(t, s)[\tau]:=\mathbb{E}_{Q_{1}}\left[\tilde{\Lambda}_{t}^{s}[\tau]\right], \quad \tau \in \mathcal{T}(\mathcal{H}), \quad 0 \leq s \leq t ;
$$

$\mathcal{U}(t, s)$ is a channel from $\mathcal{T}(\mathcal{H})$ into $\mathcal{T}(\mathcal{H})$. By points (2), (3), (4) of Assumption 2] for $0 \leq r \leq s \leq t, \sigma_{s} \in L^{1}\left(X, \mathcal{X}_{s}^{0}, Q_{1} ; \mathcal{T}(\mathcal{H})\right)$, we get

$$
\mathcal{U}(t, s) \circ \mathcal{U}(s, r)=\mathcal{U}(t, r), \quad \mathbb{E}_{Q_{1}}\left[\tilde{\Lambda}_{t}^{s}\left[\sigma_{s}\right] \mid \mathcal{X}_{s}^{0}\right]=\mathcal{U}(t, s)\left[\sigma_{s}\right]
$$

The quantum continual measurements is represented by the operators $\tilde{\Lambda}_{t}^{s}$, in the sense that they give probabilities and state changes. If $\eta_{0} \in \mathcal{S}(\mathcal{H})$ is the initial state at time 0 and $B \in \mathcal{X}_{t}^{0}$ is any event involving the output in the interval $(0, t)$, then $\int_{B} \operatorname{Tr}\left\{\tilde{\Lambda}_{t}^{0}\left[\eta_{0}\right](x)\right\} Q_{1}(\mathrm{~d} x)$ is the probability of the event $B$ and $\frac{\tilde{\Lambda}_{t}^{0}\left[\eta_{0}\right](x)}{\operatorname{Tr}\left\{\tilde{\Lambda}_{t}^{0}\left[\eta_{0}\right](x)\right\}}$ is the state at time $t$, conditional on the result $x$ (the $a$ posteriori state). Instead, $\mathcal{U}(t, 0)\left[\eta_{0}\right]$ represents the state of the system at time $t$, when the results of the measurement are not taken into account (the $a$ priori state).

\section{The initial state and the measurement}

\subsection{Ensembles}

In quantum information theory, not only single states are used, but also families of quantum states with a probability law on them, called ensembles. An ensemble $\{\mu, \rho\}$ is a probability measure $\mu(\mathrm{d} y)$ on some measurable space $(Y, \mathcal{Y})$ together with a random variable $\rho: Y \rightarrow \mathcal{S}(\mathcal{H})$. Alternatively, an ensemble can be seen as a quantum/classical state of the type described in Section 1.1.1 Given an ensemble, one can introduce an average state $\bar{\rho} \in \mathcal{S}(\mathcal{H})$

$$
\bar{\rho}:=\mathbb{E}_{\mu}[\rho]=\int_{Y} \mu(\mathrm{d} y) \rho(y) ;
$$

the integrals involving trace class operators are always understood as Bochner integrals. Finally, the average relative entropy of the states $\rho(y)$ with respect to $\bar{\rho}$ is called the " $\chi$-quantity" of the ensemble:

$$
\chi\{\mu, \rho\}:=\int_{Y} \mu(\mathrm{d} y) S_{\mathrm{q}}(\rho(y) \mid \bar{\rho})=\mathbb{E}_{\mu}\left[S_{\mathrm{q}}(\rho \mid \bar{\rho})\right] .
$$


This new quantity plays an important role in the whole quantum information theory $[3,20]$ and can be thought as a measure of some kind of quantum information stored in the ensemble.

\section{$2.2 \quad$ The letter states}

Let us consider the typical setup of quantum communication theory. A message is transmitted by encoding the letters in some quantum states, which are possibly corrupted by a quantum noisy channel; at the end of the channel the receiver attempts to decode the message by performing measurements on the quantum system. So, one has an alphabet $A$ and the letters $\alpha \in A$ are transmitted with some a priori probabilities $P_{\mathrm{i}}$. Each letter $\alpha$ is encoded in a quantum state and we denote by $\rho_{\mathrm{i}}(\alpha)$ the state associated to the letter $\alpha$ as it arrives to the receiver, after the passage through the transmission channel. While it is usual to consider a finite alphabet, also general continuous parameter spaces are acquiring importance $[19,20]$.

Assumption 3. Let $\left(A, \mathcal{A}, Q_{0}\right)$ be a probability space with $(A, \mathcal{A})$ standard Borel and let $\sigma_{\mathrm{i}}$ be a normal state on $L^{\infty}\left(A, \mathcal{A}, Q_{0} ; \mathcal{L}(\mathcal{H})\right)$.

Let us set

$$
q_{\mathrm{i}}(\alpha):=\operatorname{Tr}\left\{\sigma_{\mathrm{i}}(\alpha)\right\}, \quad \rho_{\mathrm{i}}(\alpha):=\frac{\sigma_{\mathrm{i}}(\alpha)}{q_{\mathrm{i}}(\alpha)}, \quad P_{\mathrm{i}}(\mathrm{d} \alpha):=q_{\mathrm{i}}(\alpha) Q_{0}(\mathrm{~d} \alpha) ;
$$

$q_{\mathrm{i}}$ is a probability density and $\left\{P_{\mathrm{i}}, \rho_{\mathrm{i}}\right\}$ is the initial ensemble. The average state and the $\chi$-quantity of the initial ensemble are

$$
\begin{gathered}
\eta_{0}:=\mathbb{E}_{Q_{0}}\left[\sigma_{\mathrm{i}}\right]=\int_{A} P_{\mathrm{i}}(\mathrm{d} \alpha) \rho_{\mathrm{i}}(\alpha), \\
\chi\left\{P_{\mathrm{i}}, \rho_{\mathrm{i}}\right\}:=\int_{A} P_{\mathrm{i}}(\mathrm{d} \alpha) S_{\mathrm{q}}\left(\rho_{\mathrm{i}}(\alpha) \mid \eta_{0}\right) .
\end{gathered}
$$

The quantity $\chi\left\{P_{\mathrm{i}}, \rho_{\mathrm{i}}\right\}$ is known also as Holevo capacity [3,20].

\subsection{Probabilities and states derived from $\eta_{0}$}

For $0 \leq r \leq s \leq t$ we define:

$$
\eta_{t}:=\mathcal{U}(t, 0)\left[\eta_{0}\right], \quad \tilde{\sigma}_{t}^{r}:=\tilde{\Lambda}_{t}^{r}\left[\eta_{r}\right], \quad \tilde{q}_{t}^{s}:=\left\|\tilde{\sigma}_{t}^{s}\right\|_{1}, \quad \tilde{\varrho}_{t}^{r}:=\frac{\tilde{\sigma}_{t}^{r}}{\tilde{q}_{t}^{r}} .
$$

Then, $\eta_{t}$ and $\varrho_{t}^{r}(x)$ are states on $\mathcal{L}(\mathcal{H}), \tilde{q}_{t}^{s}$ is a state on $L^{\infty}\left(X, \mathcal{X}_{t}^{0}, Q_{1}\right)$ and $\tilde{\sigma}_{t}^{r}$ a state on $L^{\infty}\left(X, \mathcal{X}_{t}^{0}, Q_{1} ; \mathcal{L}(\mathcal{H})\right)$. We have also

$$
\mathbb{E}_{Q_{1}}\left[\tilde{q}_{t}^{r} \mid \mathcal{X}_{s}^{0}\right]=\tilde{q}_{s}^{r}, \quad \mathbb{E}_{Q_{1}}\left[\tilde{q}_{t}^{r} \mid \mathcal{X}_{t}^{s}\right]=\tilde{q}_{t}^{s} .
$$

Moreover, there exists a unique probability $P_{1}$ on $(X, \mathcal{X})$ such that $\left.P_{1}(\mathrm{~d} x)\right|_{\mathcal{X}_{t}^{0}}=$ $\tilde{q}_{t}^{0}(x) Q_{1}(\mathrm{~d} x)$ for all $t \geq 0$. Also $\left.P_{1}(\mathrm{~d} x)\right|_{\mathcal{X}_{t}^{s}}=\tilde{q}_{t}^{s}(x) Q_{1}(\mathrm{~d} x)$ holds. 


\subsection{The general setup}

It is useful to unify the initial distribution and the distribution of the measurement results in a unique filtered probability space. Let us set:

$$
\begin{gathered}
\Omega:=A \times X, \quad \omega:=(\alpha, x), \quad \pi_{0}(\omega):=\alpha, \quad \pi_{1}(\omega):=x, \\
\sigma_{0}:=\sigma_{\mathrm{i}} \circ \pi_{0}, \quad q_{0}:=q_{\mathrm{i}} \circ \pi_{0}=\left\|\sigma_{0}\right\|_{1}, \quad \rho_{0}:=\rho_{\mathrm{i}} \circ \pi_{0}=\frac{\sigma_{0}}{\left\|\sigma_{0}\right\|_{1}}, \\
\mathcal{F}:=\mathcal{A} \otimes \mathcal{X}, \quad Q:=Q_{0} \otimes Q_{1}, \\
\mathcal{F}_{0}:=\{B \times X: B \in \mathcal{A}\}, \quad \mathcal{F}_{t}^{s}:=\left\{A \times Y: Y \in \mathcal{X}_{t}^{s}\right\}, \\
\mathcal{F}_{t}:=\mathcal{F}_{0} \vee \mathcal{F}_{t}^{0}=\sigma\left\{B \times Y: B \in \mathcal{A}, Y \in \mathcal{X}_{t}^{0}\right\}, .
\end{gathered}
$$

By defining $\Lambda_{t}^{s}:=\mathbb{1} \otimes \tilde{\Lambda}_{t}^{s}$, we extend $\tilde{\Lambda}_{t}^{s} \quad$ to $\quad L^{1}\left(\Omega, \mathcal{F}_{s}, Q ; \mathcal{T}(\mathcal{H})\right) \simeq$ $L^{1}\left(A, \mathcal{A}, Q_{0}\right) \otimes L^{1}\left(X, \mathcal{X}_{s}^{0}, Q_{1} ; \mathcal{T}(\mathcal{H})\right) . \quad$ Similarly, we extend $\mathcal{U}(t, s)$ to $L^{1}\left(\Omega, \mathcal{F}_{s}, Q ; \mathcal{T}(\mathcal{H})\right) \simeq L^{1}\left(\Omega, \mathcal{F}_{s}, Q\right) \otimes \mathcal{T}(\mathcal{H})$. Let us also set:

$$
\begin{gathered}
\sigma_{t}:=\Lambda_{t}^{0}\left[\sigma_{0}\right], \quad \sigma_{t}^{s}:=\tilde{\sigma}_{t}^{s} \circ \pi_{1}=\Lambda_{t}^{s}\left[\eta_{s}\right], \quad q_{t}:=\left\|\sigma_{t}\right\|_{1}, \\
q_{t}^{s}:=\tilde{q}_{t}^{s} \circ \pi_{1}=\left\|\sigma_{t}^{s}\right\|_{1}, \quad \rho_{t}:=\frac{\sigma_{t}}{\left\|\sigma_{t}\right\|_{1}}, \quad \varrho_{t}^{s}:=\tilde{\varrho}_{t}^{s} \circ \pi_{1}=\frac{\sigma_{t}^{s}}{\left\|\sigma_{t}^{s}\right\|_{1}} .
\end{gathered}
$$

In the computations of the following sections we shall need various properties of the quantities we have just introduced; here we summarize such properties. Let $r, s, t$ be three ordered times: $0 \leq r \leq s \leq t$. Then, $\sigma_{t}$ and $\sigma_{t}^{s}$ are states on $L^{\infty}\left(\Omega, \mathcal{F}_{t}, Q ; \mathcal{L}(\mathcal{H})\right)$ and

$$
\begin{gathered}
\mathbb{E}_{Q}\left[q_{t} \mid \mathcal{F}_{s}\right]=q_{s}, \quad \mathbb{E}_{Q}\left[q_{t} \mid \mathcal{F}_{t}^{s}\right]=\mathbb{E}_{Q}\left[q_{t}^{r} \mid \mathcal{F}_{t}^{s}\right]=q_{t}^{s}, \\
\mathbb{E}_{Q}\left[q_{t}^{r} \mid \mathcal{F}_{s}\right]=q_{s}^{r}, \quad \mathbb{E}_{Q}\left[\sigma_{t} \mid \mathcal{F}_{t}^{s}\right]=\mathbb{E}_{Q}\left[\sigma_{t}^{r} \mid \mathcal{F}_{t}^{s}\right]=\sigma_{t}^{s}, \\
\mathbb{E}_{Q}\left[\sigma_{t} \mid \mathcal{F}_{s}\right]=\mathcal{U}(t, s)\left[\sigma_{s}\right], \quad \mathbb{E}_{Q}\left[\sigma_{t}^{r} \mid \mathcal{F}_{s}\right]=\mathcal{U}(t, s)\left[\sigma_{s}^{r}\right], \\
\mathbb{E}_{Q}\left[\sigma_{t}^{s} \mid \mathcal{F}_{s}\right]=\eta_{t}, \quad \eta_{t}=\mathbb{E}_{Q}\left[\sigma_{t}\right], \quad \sigma_{t}=\Lambda_{t}^{s}\left[\sigma_{s}\right], \\
\sigma_{t}^{r}=\Lambda_{t}^{s}\left[\sigma_{s}^{r}\right], \quad \frac{\Lambda_{t}^{s}\left[\rho_{s}\right]}{\left\|\Lambda_{t}^{s}\left[\rho_{s}\right]\right\|_{1}}=\rho_{t}, \quad \frac{\Lambda_{t}^{s}\left[\varrho_{s}^{r}\right]}{\left\|\Lambda_{t}^{s}\left[\varrho_{s}^{r}\right]\right\|_{1}}=\varrho_{t}^{r} .
\end{gathered}
$$

We have that $\left\{q_{t}, t \geq 0\right\}$ is a non-negative, mean one, $Q$-martingale. Then, there exists a unique probability $P$ on $(\Omega, \mathcal{F})$ such that $\forall t \geq 0$

$$
\left.P(\mathrm{~d} \omega)\right|_{\mathcal{F}_{t}}=q_{t}(\omega) Q(\mathrm{~d} \omega) .
$$

Moreover,

$$
\begin{array}{cl}
P(\mathrm{~d} \alpha \times X)=P_{\mathrm{i}}(\mathrm{d} \alpha), & P(A \times \mathrm{d} x)=P_{1}(\mathrm{~d} x), \\
\left.P(\mathrm{~d} \omega)\right|_{\mathcal{F}_{t}^{s}}=q_{t}^{s}(\omega) Q(\mathrm{~d} \omega), & \eta_{t}=\mathbb{E}_{P}\left[\rho_{t}\right]=\mathcal{U}(t, s)\left[\eta_{s}\right] .
\end{array}
$$

\section{Mutual entropies and informational bounds}

Here and in the following we shall have always $0 \leq u \leq r \leq s \leq t$. 


\subsection{The state $q_{t}$ and the classical information}

Let us consider the state $q_{t}$ and its marginals $\mathbb{E}_{Q}\left[q_{t} \mid \mathcal{F}_{r}\right]=q_{r}, \mathbb{E}_{Q}\left[q_{t} \mid \mathcal{F}_{t}^{r}\right]=q_{t}^{r}$.

Then, we can introduce the classical mutual entropy:

$$
S_{\mathrm{c}}\left(q_{t} \mid q_{r} q_{t}^{r}\right)=\int_{\Omega} P(\mathrm{~d} \omega) \log \frac{q_{t}(\omega)}{q_{r}(\omega) q_{t}^{r}(\omega)}=: I_{\mathrm{c}}(r, t) .
$$

Note that $I_{\mathrm{c}}(t, t)=0$. For $r=0$ we have the input/output classical information gain:

$$
I_{\mathrm{c}}(0, t)=S_{\mathrm{c}}\left(q_{t} \mid q_{\mathrm{i}} \otimes \tilde{q}_{t}^{0}\right) \equiv \int_{A \times X} P(\mathrm{~d} \alpha \times \mathrm{d} x) \log \frac{q_{t}(\alpha, x)}{q_{\mathrm{i}}(\alpha) \tilde{q}_{t}^{0}(x)} .
$$

By applying the monotonicity theorem and the channel $\mathbb{E}_{Q}\left[\bullet \mid \mathcal{F}_{s}\right]$ to the couple of states $q_{t}$ and $q_{r} q_{t}^{r}$, we get

$$
S_{\mathrm{c}}\left(q_{t} \mid q_{r} q_{t}^{r}\right) \geq S_{\mathrm{c}}\left(\mathbb{E}_{Q}\left[q_{t} \mid \mathcal{F}_{s}\right] \mid \mathbb{E}_{Q}\left[q_{r} q_{t}^{r} \mid \mathcal{F}_{s}\right]\right)=S_{\mathrm{c}}\left(q_{s} \mid q_{r} q_{s}^{r}\right),
$$

which becomes

$$
I_{\mathrm{c}}(r, t) \geq I_{\mathrm{c}}(r, s) .
$$

The function $t \mapsto I_{\mathrm{c}}(s, t)$ is non decreasing.

\subsection{The state $\sigma_{s}$ and the main bound}

A useful quantity, with the meaning of a measure of the "quantum information" left in the a posteriori states, is the mean $\chi$-quantity

$$
\bar{\chi}(s, t):=\int_{\Omega} P(\mathrm{~d} \omega) S_{\mathrm{q}}\left(\rho_{t}(\omega) \mid \varrho_{t}^{s}(\omega)\right)=\mathbb{E}_{P}\left[S_{\mathrm{q}}\left(\rho_{t} \mid \varrho_{t}^{s}\right)\right] .
$$

The interpretation as a mean $\chi$-quantity is due to the fact that $\bar{\chi}(s, t)=$ $\mathbb{E}_{P}\left[\mathbb{E}_{P}\left[S_{\mathrm{q}}\left(\rho_{t} \mid \varrho_{t}^{s}\right) \mid \mathcal{F}_{t}^{s}\right]\right]$. But by Eq. (77) and $\mathbb{E}_{P}\left[\rho_{t} \mid \mathcal{F}_{t}^{s}\right]=\varrho_{t}^{s}, \mathbb{E}_{P}\left[\varrho_{t}^{s} \mid \mathcal{F}_{t}^{s}\right]=\varrho_{t}^{s}$, we have that $\mathbb{E}_{P}\left[S_{\mathrm{q}}\left(\rho_{t} \mid \varrho_{t}^{s}\right) \mid \mathcal{F}_{t}^{s}\right]$ is a random $\chi$-quantity. Note that

$$
\bar{\chi}(t, t)=\int_{\Omega} P(\mathrm{~d} \omega) S_{\mathrm{q}}\left(\rho_{t}(\omega) \mid \eta_{t}\right)=: \chi\left\{P, \rho_{t}\right\} .
$$

Let us consider the state $\sigma_{s}$ and its marginals $\mathbb{E}_{Q}\left[\operatorname{Tr}\left\{\sigma_{s}\right\} \mid \mathcal{F}_{r}\right]=q_{r}$, $\mathbb{E}_{Q}\left[\sigma_{s} \mid \mathcal{F}_{s}^{r}\right]=\sigma_{s}^{r}$. Then, we have the mutual entropy

$$
S\left(\sigma_{s} \mid q_{r} \sigma_{s}^{r}\right)=I_{\mathrm{c}}(r, s)+\bar{\chi}(r, s) .
$$

For $r=s$ and for $r=s=0$ this equation reduces to

$$
S\left(\sigma_{s} \mid q_{s} \eta_{s}\right)=\chi\left\{P, \rho_{s}\right\}, \quad S\left(\sigma_{0} \mid q_{0} \eta_{0}\right)=\chi\left\{P, \rho_{0}\right\}=\chi\left\{P_{\mathrm{i}}, \rho_{\mathrm{i}}\right\} .
$$

By applying the monotonicity theorem and the channel $\Lambda_{t}^{s}$ to the couple of states $\sigma_{s}$ and $q_{r} \sigma_{s}^{r}$, we get

$$
S\left(\sigma_{s} \mid q_{r} \sigma_{s}^{r}\right) \geq S\left(\Lambda_{t}^{s}\left[\sigma_{s}\right] \mid \Lambda_{t}^{s}\left[q_{r} \sigma_{s}^{r}\right]\right)=S\left(\sigma_{t} \mid q_{r} \sigma_{t}^{r}\right),
$$

which becomes

$$
\bar{\chi}(r, s)-\bar{\chi}(r, t) \geq I_{\mathrm{c}}(r, t)-I_{\mathrm{c}}(r, s) \geq 0 .
$$


Therefore, the function $t \mapsto \bar{\chi}(s, t)$ is non increasing.

For $r=s$ we get

$$
S\left(\sigma_{s} \mid q_{s} \eta_{s}\right) \geq S\left(\sigma_{t} \mid q_{s} \sigma_{t}^{s}\right),
$$

which gives the upper bound for $I_{\mathrm{C}}$ :

$$
0 \leq I_{\mathrm{c}}(s, t) \leq \chi\left\{P, \rho_{s}\right\}-\bar{\chi}(s, t) .
$$

For $s=r=0$, it reduces to

$$
0 \leq I_{\mathrm{c}}(0, t) \leq \chi\left\{P_{\mathrm{i}}, \rho_{\mathrm{i}}\right\}-\int_{A \times X} P(\mathrm{~d} \alpha \times \mathrm{d} x) S_{\mathrm{q}}\left(\rho_{t}(\alpha, x) \mid \tilde{\varrho}_{t}^{0}(x)\right) .
$$

The bound (30) is the translation in terms of continual measurements of the bound of Section 3.3.4 of [7], which in turn is a generalization of a bound by Schumacher, Westmoreland and Wootters [5]. Equation (30) is a strengthening of the Holevo bound [3] $I_{\mathrm{c}}(0, t) \leq \chi\left\{P_{\mathrm{i}}, \rho_{\mathrm{i}}\right\}$.

\subsection{Quantum information gain}

Let us consider now the quantum information gain defined by the quantum entropy of the pre-measurement state minus the mean entropy of the a posteriori states $[1,2,4]$. It is a measure of the gain in purity (or loss, if negative) in passing from the pre-measurement state to the post-measurement a posteriori states. In the continual case, we can consider the quantum information gain in the time interval $(s, t)$ when the system is prepared in the ensemble $\left\{P_{\mathrm{i}}, \rho_{\mathrm{i}}\right\}$ at time 0 or when it is prepared in the state $\eta_{r}$ at time $r$ :

$$
\begin{gathered}
I_{\mathrm{q}}(s, t):=\int_{\Omega} P(\mathrm{~d} \omega)\left[S_{\mathrm{q}}\left(\rho_{s}(\omega)\right)-S_{\mathrm{q}}\left(\rho_{t}(\omega)\right)\right], \\
I_{\mathrm{q}}(r ; s, t):=\int_{\Omega} P(\mathrm{~d} \omega)\left[S_{\mathrm{q}}\left(\varrho_{s}^{r}(\omega)\right)-S_{\mathrm{q}}\left(\varrho_{t}^{r}(\omega)\right)\right] .
\end{gathered}
$$

By this definition we have immediately

$$
I_{\mathrm{q}}(r, t)=I_{\mathrm{q}}(r, s)+I_{\mathrm{q}}(s, t), \quad I_{\mathrm{q}}(u ; r, t)=I_{\mathrm{q}}(u ; r, s)+I_{\mathrm{q}}(u ; s, t) .
$$

It has been proved [4] that the quantum information gain is positive for all initial states if and only if the measurement sends pure initial states into pure a posteriori states.

As in the single time case [6-8], inequality (27) can be easily transformed into an inequality involving $I_{\mathrm{q}}$ :

$$
I_{\mathrm{q}}(r ; s, t)-I_{\mathrm{q}}(s, t) \geq I_{\mathrm{c}}(r, t)-I_{\mathrm{c}}(r, s) \geq 0 .
$$

Let us take an initial ensemble made up of pure states: $\rho_{\mathrm{i}}(\alpha)^{2}=\rho_{\mathrm{i}}(\alpha)$, $\forall \alpha \in A$. Let us assume that the continual measurement preserve pure states: the states $\rho_{t}(\alpha, x)$ are pure for all choices of $t, \alpha, x$. Then, the von Neumann entropy of $\rho_{t}(\omega)$ vanishes and we have $I_{\mathrm{q}}(s, t)=0$ for all choices of $s$ and $t$. From the second of Eqs. (32) and Eq. (33) we get

$$
I_{\mathrm{q}}(u ; r, t)-I_{\mathrm{q}}(u ; r, s)=I_{\mathrm{q}}(u ; s, t) \geq I_{\mathrm{c}}(u, t)-I_{\mathrm{c}}(u, s) \geq 0,
$$


i.e. the function $t \mapsto I_{\mathrm{q}}(u ; r, t)$ is non decreasing for "pure" continual measurements.

In particular, by taking $u=r=0$ we have

$$
I_{\mathrm{q}}(0 ; 0, t)=S_{\mathrm{q}}\left(\eta_{0}\right)-\int_{X} P_{1}(\mathrm{~d} x) S_{\mathrm{q}}\left(\tilde{\varrho}_{t}^{0}(x)\right) .
$$

For a continual measurement sending every pure initial state into pure a posteriori states, $\forall \eta_{0} \in \mathcal{S}(\mathcal{H})$ the quantum information gain $I_{\mathrm{q}}(0 ; 0, t)$ is non negative, non decreasing in time and with $I_{\mathrm{q}}(0 ; 0,0)=0$.

\section{Acknowledgments}

Work supported by the European Community's Human Potential Programme under contract HPRN-CT-2002-00279, QP-Applications, and by Istituto Nazionale di Fisica Nucleare.

\section{References}

[1] H. J. Groenewold, A problem of information gain by quantal measurements, Int. J. Theor. Phys. 4 (1971) 327-338.

[2] G. Lindblad, An entropy inequality for quantum measurements, Commun. Math. Phys. 28 (1972) 245-249.

[3] A. S. Holevo, Some estimates for the amount of information transmittable by a quantum communication channel, Probl. Inform. Transm. 9 no. 3 (1973) 177-183 (Engl. transl.: 1975).

[4] M. Ozawa, On information gain by quantum measurements of continuous observables, J. Math. Phys. 27 (1986) 759-763.

[5] B. Schumacher, M. Westmoreland, and W. K. Wootters, Limitation on the amount of accessible information in a quantum channel, Phys. Rev. Lett. 76 (1996) 3452-3455.

[6] A. Barchielli and G. Lupieri, Instruments and channels in quantum information theory, Optics and Spectroscopy 99 (2005) 425-432; quant-ph/0409019

[7] A. Barchielli and G. Lupieri, Instruments and mutual entropies in quantum information theory, to appear in Banach Center Publications; quant-ph/0412116

[8] A. Barchielli and G. Lupieri, Quantum measurements and entropic bounds on information transmission, to appear in Quantum Inform. Compu.; quant-ph/0505090

[9] A. Barchielli, Entropy and information gain in quantum continual measurements, in P. Tombesi and O. Hirota (eds.), Quantum Communication, Computing, and Measurement 3 (Kluwer, New York, 2001) pp. 49-57; quant-ph/0012115

[10] A. Barchielli and G. Lupieri, Instrumental processes, entropies, information in quantum continual measurements, in O. Hirota (ed.), Quantum Information, Statistics, Probability (Rinton, Princeton, 2004) pp. 30-43; Quantum Inform. Compu. 4 (2004) 437-449; quant-ph/0401114 
[11] S. Sakai, $C^{*}$-Algebras and $W^{*}$-Algebras (Springer, Berlin, 1971).

[12] M. Ohya and D. Petz, Quantum Entropy and Its Use (Springer, Berlin, 1993).

[13] J. Dixmier, Les Algèbres d'Opérateurs dans l'Espace Hilbertien (GauthierVillars, Paris, 1957).

[14] A. S. Holevo, Statistical Structure of Quantum Theory, Lect. Notes Phys. m67 (Springer, Berlin, 2001).

[15] E. B. Davies, Quantum Theory of Open Systems (Academic Press, London, 1976).

[16] M. Ozawa, Quantum measuring processes of continuous observables, J. Math. Phys. 25 (1984) 79-87.

[17] M. Ozawa, Conditional probability and a posteriori states in quantum mechanics, Publ. R.I.M.S. Kyoto Univ. 21 (1985) 279-295.

[18] A. Barchielli, Stochastic processes and continual measurements in quantum mechanics, in S. Albeverio, G. Casati, D. Merlini (eds.), Stochastic Processes in Classical and Quantum Systems. LNP 262 (Springer, Berlin, 1986), pp. 14-23.

[19] H. P. Yuen and M. Ozawa, Ultimate information carrying limit of quantum systems, Phys. Rev. Lett. 70 (1993) 363-366.

[20] A. S. Holevo, M. E. Shirokov, Continuous ensembles and the $\chi$-capacity of infinite dimensional channels, quant-ph/0408176. 